\title{
Compruebe sus conocimientos sobre las técnicas de planifícación (I)
}

Margarita Peya Gascóns

Profesora titular. Administración Servicios de Enfermería. Escuela de Enfermería de la Universidad de Barcelona. Barcelona. España.

as llamadas técnicas de planificación sanitaria son reconocidas por su utilidad en el ámbito de los trabajos de grupo y en la realización de estudios. Por ejemplo, el brainstorming es muy utilizado para la detección de problemas en la mejora de la calidad asistencial. El Grupo Nominal se suele aplicar en las situaciones o casos que queremos mejorar basándonos en la reflexión y en el consenso de un grupo de participantes. Por otra parte, la técnica de Delphi permite ver tendencias de futuro, de acuerdo, por ejemplo, con la visión de los profesionales de la salud y los usuarios. El diagrama causa-efecto de Ishikawa puede ayudarnos a delimitar los problemas ante un acontecimiento determinado. A continuación le presentamos una serie de preguntas cuyas respuestas le darán a conocer su nivel de conocimientos sobre estas útiles técnicas.

1. La técnica de brainstorming también es llamada:

a. Lluvia de ideas.

b. Remueva meninges.

c. Tormenta de ideas.

d. Todas son válidas.

2. El brainstorming es una técnica concebida para:

a. Emitir ideas en el seno de un grupo.

b. Clasificar problemas por orden de importancia.

c. Resolver problemas mediante la reflexión individual.

d. b y c son ciertas.

3. El brainstorming es una técnica de planificación útil para:

a. Priorizar los problemas detectados.

b. Detectar problemas relativos a una situación.

c. Buscar alternativas para resolver problemas.

d. b y c son ciertas.

4. Los participantes en la realización de una técnica de brainstorming:

a. Aportan el máximo de ideas posibles, de forma razonada y justificada.

b. Es obligatorio que todos aporten el mismo número de ideas.

c. Emiten el máximo de ideas, de la forma más rápida posible, sin justificar y sin argumentar.

d. Es importante que se voten siempre las ideas para llegar al máximo consenso.
5. La llamada fase de explotación de un brainstorming responde a:

a. Una fase de calma intermedia para reflexionar sobre las ideas que se están emitiendo.

b. Una fase de calma al final de la emisión de ideas en que se analizan, se clasifican y se reformulan dichas ideas.

c. Una fase de calma intermedia para eliminar ideas que no se corresponden con el tema que se va a analizar.

d. Una fase crítica de priorización de ideas.

6. Algunos de los problemas que dificultan la realización de un brainstorming son:

a. La timidez y la baja autoestima.

b. El miedo a la crítica y a hacer el ridículo.

c. La rigidez del moderador.

d. Todas son ciertas.

7. El objetivo de la técnica del Grupo Nominal (Delbec) es:

a. La emisión de ideas de forma creativa.

b. La identificación de problemas y su clasificación por orden de importancia.

c. La priorización de problemas de forma individual y no presencial.

d. a y c son ciertas.

8. La técnica del Grupo Nominal (Delbec) es un método de consenso que se basa en:

a. La elaboración de cuestionarios que se remiten por correo. b. La generación de ideas por medio de la reflexión individual y en la evaluación en base al consenso.

c. El análisis interno y externo de una organización.

d. Delimitar los problemas sobre una situación y descubrir sus causas.

9. Algunas de las etapas de la técnica del Grupo Nominal (Delbec) son:

a. La generación individual de ideas, su redacción en silencio y un período grupal de discusión y clarificación.

b. La votación primero en silencio y después en discusión para lograr el consenso y la votación final.

c. La ausencia de discusión e intercambio directo de ideas.

d. a y b son ciertas

10. Las ventajas de la utilización de la técnica del Grupo Nominal (Delbec) son que:

a. Es un método muy preciso.

b. Da la oportunidad a los participantes

de expresar sus opiniones.

c. Produce un gran número de ideas.

d. b y c son ciertas.

11. El objetivo de la técnica

Delphi es:

a. Describir la magnitud y la gravedad de un problema.

b. Facilitar la comunicación entre los participantes. 
c. Conocer la opinión de las personas sobre temas prevalentes y tendencias de futuro.

d. a y b son ciertas.

12. La técnica de Delphi como método de consenso se basa en:

a. El diseño de diferentes flechas que indican las actividades que se van a realizar.

b. La elaboración de cuestionarios que se envían por correo.

c. Formas gráficas que muestran los problemas.

d. La generación de ideas a partir de la reflexión individual presencial.

13. Una de las principales ventajas de la técnica Delphi es:

a. El anonimato.

b. Disponer de tiempo para la reflexión.

c. No requiere que los participantes se desplacen.

d. Todas son ciertas.

14. El diagrama causa-efecto de Ishikawa recibe también el nombre de:

a. Diagrama en árbol.

b. Diagrama de barras.

c. Diagrama en espina de pescado.

d. Diagrama de círculos.

15. El objetivo el diagrama causaefecto de Ishikawa es:

a. Es un diagrama de barras que muestra los períodos de inicio y final de las actividades de un proyecto.

b. Diagrama que busca la eficacia de las soluciones a problemas.

c. Diagrama que muestra los resultados que se pretenden lograr en un tiempo determinado.

d. Diagrama que delimita los problemas, sus causas y facilita la comunicación.

16. El diagrama causa-efecto de Ishikawa se compone de:

a. Diferentes flechas: una grande horizontal que indica el resultado deseado y flechas oblicuas principales y suplementarias que muestran las actividades.

b. Diferentes barras que indican el cronograma de un proyecto.

c. Círculos y cuadrados que indican el procedimiento que se va a seguir.

d. Todas son ciertas

\section{Respuestas}

1 d. La técnica de brainstorming se conoce por estos distintos nombres. 2 a. El brainstorming está concebido para estimular la emisión de ideas y se basa en la creencia de que toda persona es capaz de innovar, de inventar y de crear. Clasificar problemas por orden de importancia y resolver problemas mediante la reflexión individual son características del método del Grupo Nominal (Delbec).

3 d. Detectar problemas relativos a una situación y buscar alternativas para resolver problemas son dos de las utilidades de esta técnica de brainstorming. Priorizar los problemas responde a las escalas de priorización y otras técnicas de planificación.

4 c. Uno de los objetivos del brainstorming es que los participantes rompan las limitaciones habituales del pensamiento y generen una gran cantidad de ideas, sin razonar ni justificar. Tampoco es obligado que los participantes aporten ideas cada vez. Y no siempre es necesario votar para llegar al consenso.

$\mathbf{5} \mathbf{b}$. En la técnica de brainstorming, al terminar la emisión de ideas se inicia la fase de explotación o calma, en la que los participantes analizan, clasifican, agrupan y reformulan las ideas. No existen fases de calma intermedia, ya que romperían la emisión de ideas, y tampoco es una característica del brainstorming la priorización de ideas o de problemas.

$6 \mathbf{d}$. Todos son aspectos que pueden dificultar la realización de esta técnica de brainstorming.

7 b. Es el principal objetivo de la técnica del Grupo Nominal (Delbec). La respuesta a responde al brainstorming y la c corresponde a una de las etapas de la técnica Delphi.

8 b. El fundamento del método nominal (Delbec) es generar ideas a partir de la reflexión individual de la persona y la evaluación para alcanzar el consenso. La respuesta a corresponde a una de las etapas del método Delphi. La respuesta c hace referencia a una técnica de planificación estratégica (CAME-DAFO) y la $d$ responde al diagrama de Ishikawa.

9 d. La reflexión individual y la discusión en grupo para clarificar y lograr consenso son características de las etapas de la técnica del Grupo Nominal (Delbec). La respuesta $c$ responde a la técnica Delphi. 10 d. La ocasión que tienen los participantes de expresar sus ideas y su gran producción son ventajas de esta técnica del Grupo Nominal (Delbec) La falta de precisión es uno de sus inconvenientes.

11 c. La técnica Delphi es uno de los métodos más utilizados para conocer los temas de prevalencia y las tendencias que puede deparar el futuro con respecto a temas previamente determinados. $\mathrm{La}$ respuesta a responde a características del método Hanlon. La $b$ responde a cualquier técnica de planificación presencial.

12 b. Una de las características de la técnica Delphi es la no presencia de los participantes, a los que se les requiere que respondan diferentes cuestionarios que les son remitidos por correo. La respuesta a corresponde al diagrama Ishikawa. La c, a cualquier diagrama, por ejemplo el de barras. La $d$ hace referencia al grupo nominal (Delbec).

$13 \mathbf{d}$. Todas son ventajas de la técnica Delphi.

14 c. Éste es el nombre con que se le suele conocer en nuestro país, por la estructura que tiene.

15 d. Un diagrama que facilita el análisis de problemas y sus efectos.

16 a. Representación gráfica sencilla que muestra una flecha principal y otras secundarias. La respuesta $b$ corresponde al diagrama de Gantt, y la c, a un diagrama de flujo. 상

\section{Bibliografía general}

Asua Batarrita J. Entre el consenso y la evidencia científica. Gac Sanit. 2005;19(1).

Huglo PE. Les cercles de qualité à l'hôpital. París Berger-Levarault; 2002.

Martínez, M. Componentes ADO en Delphi. Referencia rápida. Disponible en $<\mathrm{http}$ ://www. egroups.com/group/delphidf.htm>

Monteil B, Perigord M, Raveleau G. Les outils des cercles et de l'amélioration de la qualité. París: Les Editions d'Organisation Management 2000; 1999.

Pineault R, Daveluy C. La planificación sanitaria. Conceptos, métodos y estrategias. Barcelona: Masson; 1989

Varo J. Gestión estratégica de la calidad en los servicios sanitarios. Madrid: Díaz de Santos; 1994

Correo electrónico: mpeya@ub.edu 